\title{
Kontrollmechanismen der Glycolyse
}

\author{
Benno Hess und Karl Brand \\ Max-Planck-Institut für Ernäbrungsphysiologie, Dortmund
}

\begin{abstract}
Control mechanisms of glycolysis. Control mechanisms of enzymic reactions are generally based on interactions between activators, inhibitors, substrates and products with enzyme proteins or on induction and repression of enzyme synthesis. All main types of control can be recognized in glycolysis. They are the basis of the network which controls the over-all glycolytic function and operates according to the feed-back principle. - Enzyme profiles may not be used for a functional definition of the metabolic state. Enzyme activities are governed by a variety of control mechanisms, which can best be recognized by steadystate and transient state analysis of metabolites and by an analysis of the system's response in titration experiments with pure enzymes under conditions whereby the system displays oscillatory behaviour of its over-all flux. The important parameter for the definition of the metabolic state is the net-flux through the system, since this parameter, along with the steadystate levels of the meabolites, gives the steady-state flux pattern, reveals the linetic state of enzymic reactions and points to control points of metabolism. Continuous glycolytic oscillations in a cell-free extract of Saccharomyces carlsbergensis have been observed over a period of 22 hours with a constant frequency of $0.17 \mathrm{~min}^{-1}$ and a rate of $7.2 \mathrm{nMol}$ ethanol per $\mathrm{mg}$ protein per min. Titration of such an extract by pure yeast enzymes reveals the gain (FDP, ADP) and damping components (ATP), which are fed back to the enzymes PFK and PK, respectively, PFK, PGK and PK operating as the control units. On the basis of the titration data as well as metabolite and enzyme activity phase relationship, the mechanism of this oscillation can be understood as a crossed feed-back interaction. Furthermore, it is discussed as the biochemical model of a physiological clock mechanism.
\end{abstract}

\section{EINLEITUNG}

Mit der Kristallisation des letzten nicht kristallisierten Gärungsenzyms, der Phosphofruktokinase, durch Krebs und seine Mitarbeiter (PARMiggiann,, Love \& KREBS 1964) kann die klassische Periode der Erforschung der Glycolyse als abgeschlossen betrachtet werden. Unser heutiges Interesse richtet sich auf zwei verschiedene Gesichtspunkte der Glycolyse, einmal auf die elementaren Reaktionsmechanismen der Katalyse am Enzym, einer neuen Chemie der Übergangszustände (EIGEN \& Hammes 1963), zweitens auf die Kontrollmechanismen, die den Ablauf der einzelnen Reaktionsschritte der Glycolyse bestimmen (Hess \& Chance 1959, Chance \& Hess 1959). Als Grundlage der Diskussion sollen zunächst formale Gesichtspunkte der Kontrolle enzymatischer Reaktionen erörtert werden; dann will ich den stationären Zustand sowie ein mathematisches Modell der Glycolyse und schließlich einen neuen oszillatorischen Übergangszustand der Glycolyse besprechen. 


\section{KONTROLLMECHANISMEN ENZYMATISCHER REAKTIONEN}

In Tabelle 1 sind allgemeine Kontrollmechanismen enzymatischer Reaktionen mit Beispielen aus der glycolytischen Enzymsequenz zusammengestellt. Wir unterscheiden zwei Typen der Kontrolle enzymatischer Wirksamkeiten, die sich aus der allgemeinen Umsatzgleichung enzymatischer Reaktionen ergeben: (1) Kontrolle von Enzymaktivitäten und (2) Kontrolle von Enzymkonzentrationen (Hess \& Brand 1965, Hess 1963).

Tabelle 1

Kontrollmechanismen glykolytischer Enzyme. (Nach Hess 1963 und Hess \& BRAND 1965)

\begin{tabular}{|c|c|c|}
\hline Typ & Mechanismus" & Beispiele \\
\hline $\begin{array}{l}\text { A. Kontrolle der Enzymaktivität } \\
\text { 1. Energiekontrolle durch } \\
\text { Massenwirkung } \\
\text { Autokontrolle }\end{array}$ & $\begin{array}{l}\text { Substrataktivierung } S+E \rightarrow S E \\
\text { Substrathemmung } \mathrm{S}+\mathrm{E} \rightarrow \mathrm{SE} \\
\text { Produktaktivierung } \mathrm{P}+\mathrm{E} \rightarrow \mathrm{PE} \\
\text { Produkthemmung } \mathrm{P}+\mathrm{E} \rightarrow \mathrm{PE} \\
\text { Aktivierung und Hemmung von } \\
\text { Enzymen durd gemeinsame } \\
\text { Substrate und/oder Produkte } \\
\mathrm{S}+\mathrm{E}_{1}+\mathrm{E}_{2} \rightarrow \mathrm{SE}_{1}+\mathrm{SE}_{2}\end{array}$ & $\begin{array}{l}\text { ENO, GAPDH, } \\
\text { GAPDH } \\
\text { PFK } \\
\text { GAPDH } \\
\text { PGK, PK } \\
\text { GAPDH, LDH } \\
\text { ADH, u. a. }\end{array}$ \\
\hline 2. Katalytische Kontrolle & $\begin{array}{cc}\text { Aktivierung } & \mathrm{A}+\mathrm{E} \rightarrow \mathrm{AE} \\
& 2 \mathrm{~A}+\mathrm{E} \rightarrow \mathrm{A}_{2} \mathrm{E} \\
\text { Hemmung } & \mathrm{etc} \\
& \mathrm{I}+\mathrm{E} \rightarrow \mathrm{IE} \\
& \begin{aligned} & \mathrm{E} \rightarrow \mathrm{I}_{2} \mathrm{E} \\
& \text { etc. }\end{aligned}\end{array}$ & PFK, PK \\
\hline $\begin{array}{l}\text { B. Kontrolle der Enzymkonzentrati } \\
\text { Induktion der Enzymsynthese } \\
\text { Repression der Enzymsynthese } \\
\text { Katabolische Repression }\end{array}$ & $\begin{array}{l}\mathrm{S}_{\mathrm{A}} \rightarrow{ } \mathrm{GEN} " \rightarrow \mathrm{E}_{\mathrm{A}} \\
\mathrm{PP}_{\mathrm{P}} \rightarrow \text { GEN" } \rightarrow \mathrm{E}_{\mathrm{A}} \\
\mathrm{S}_{\mathrm{A}} \rightarrow \mathrm{GEN} \rightarrow \mathrm{E}_{\mathrm{X}}\end{array}$ & $\begin{array}{l}\text { PDC } \\
? \\
\text { MDH/LDH }\end{array}$ \\
\hline \multicolumn{3}{|c|}{ 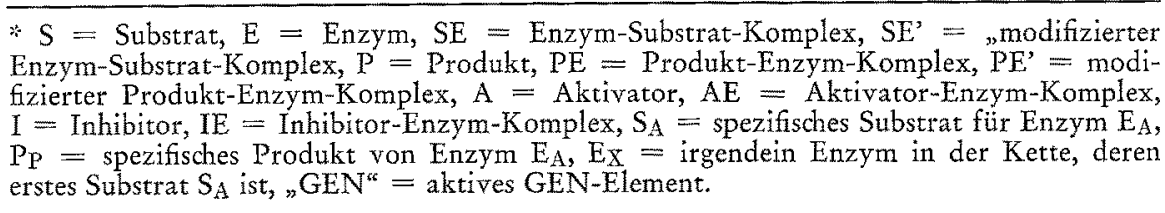 } \\
\hline
\end{tabular}

Die Kontrolle von Enzy maktivitäten wird gewöhnlich mit Übergangszeiten von Sekunden realisiert und ist für die rasche Anpassung des Stoffwechsels an neue Bedingungen verantwortlich zu machen. Sie kann durch einfache Massenwirkung (Energiekontrolle), sei es durch Substrat beziehungsweise Produktwirkung, oder durch Competition mehrerer Enzyme um gemeinsame Metaboliten zustande kommen und bildet das Beispiel der älteren Kontrolltheorien des Stoffwechsels auf der Grundlage stöchiometrischer Wechselwirkungen.

Weitaus wirksamer dïrfte der zweite Kontrollmechanismus durch katalytische Wechselwirkung mit Enzymen sein. Hier tritt die Kontrollsubstanz [ein Aktivator(A) 
oder ein Inhibitor (I)] im Sinne eines chemischen Signals zu informativer Koppelung mit dem Enzym unter Konformationsänderung in Reaktion. Entscheidend ist, daß die Substanz dabei nicht verbraucht wird. Die allosterische Aktivierung oder Hemmung sind ein Beispiel für diesen Mechanismus (Monod, Changeux \& JACOB 1963).

Der Uber-alles-Mechanismus der Kontrolle von Enz ymkonzentration wird durch Induktion und Repressionsphänomene beschrieben. Derartige Kontrollwirkungen nehmen den Apparat der Eiweißsynthese in Anspruch und haben Ubergangszeiten bis zu Stunden. Für unsere Betrachtungen wollen wir diese Mechanismen vernachlässigen.

\section{GARUNG}

Trägt man die Wechselwirkung glycolytischer Intermediate in ein qualitatives Flußdiagramm ein (Abb. 1), so tritt bei der engen inneren Vernetzung der gesamten Stoffwechselkette das Prinzip der Rückkopplung oder auch Vorwärtskopplung klar hervor, wenn auch zunächst zwischen energetischer und informativer Kopplung nicht

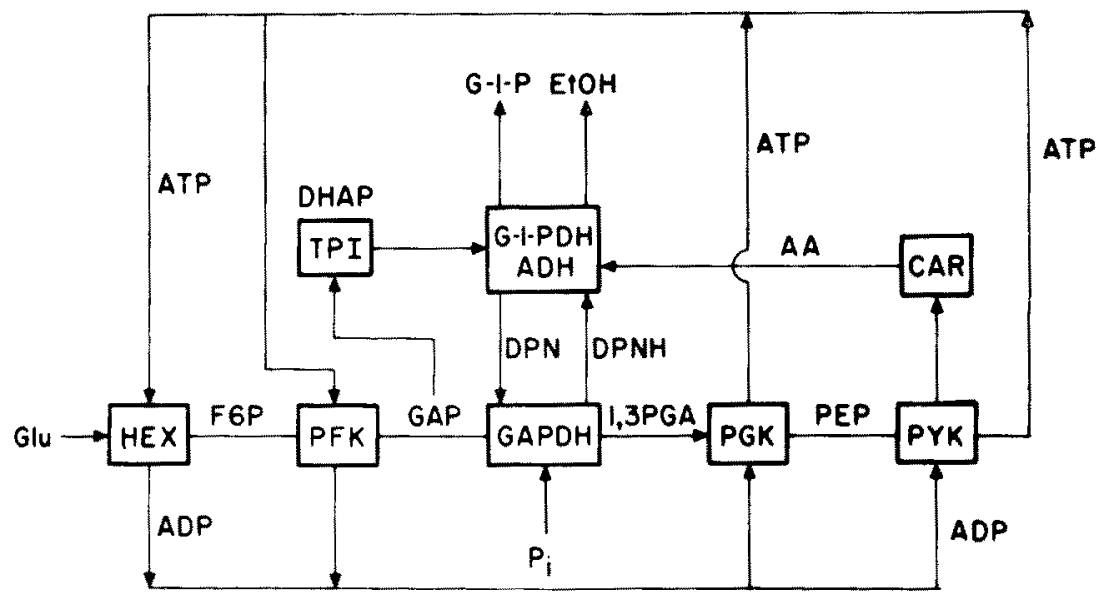

Abb. 1: Vereinfachtes Fluß̉iagramm der Gärung

unterschieden werden kann. Energetische Rückkopplung ist starr wie ein Getriebe, informative Rückkopplung ist integrativ. Durch Eingriff an mehreren Punkten der Kette (multiside control) kann der Fluß durch das System nicht nur durch schrittweise Massenwirkung, sondern durch Aktivierungs- und Zügelungskontrolle jeweils um ein Vielfaches positiv oder negativ beeinflußt werden (siehe unten). Durch die Wirkung mehrerer Kontrollmetaboliten an mehreren Stellen (concerted action) wird die Kontrolle des glycolytischen Flusses über die ganze Kette koordiniert. Die Kontrollenzyme (insbesondere die Kinasen) werden durch einen Mechanismus aktiviert, der sie funktionell zu biochemischen "Schaltern" des Zellstoffwechsels macht. Von speziellem Interesse ist in manchen glycolytischen Systemen die mögliche Flußumkehr mit Hilfe von Gegenreaktionen eigener "glycosynthetischer" Enzyme, die prinzipiell in Symme- 
trie zu den Kontrollenzymen der Glycolyse zur Überbrückung thermodynamischer Gefälle angelegt sind (zum Beispiel FDPase).

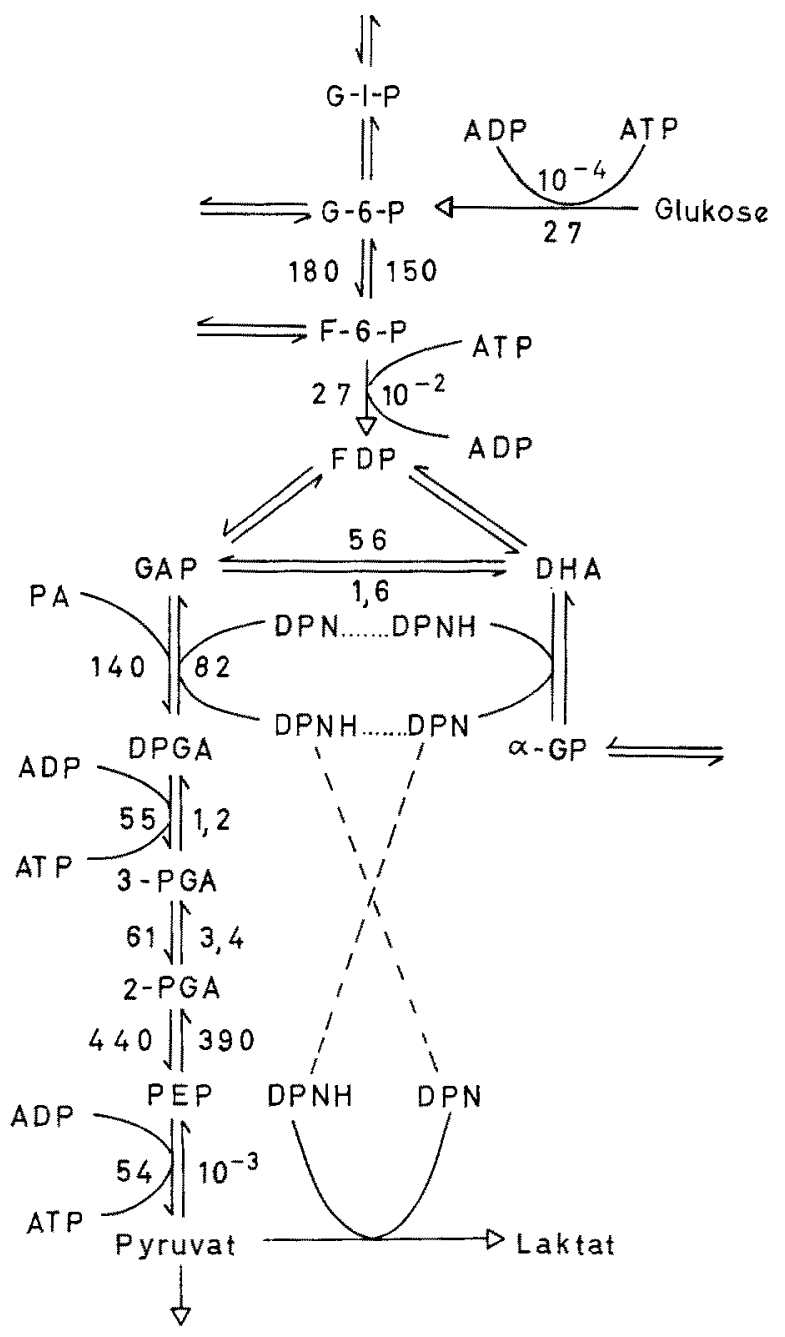

Abb. 2: Flußdiagramm der Glykolyse von Ascites-Tumorzellen, die Ziffern geben den Umsatz in $\mu \mathrm{M} \cdot \sec ^{-1}$ (nach Hess, 1963) an

\section{PROFILE GLYCOLYTISCHER FLIESSGLEICHGEWICHTE}

PETTE (1965) hat durch die Bestimmung glycolytischer Enzymverteilungsmuster in einer großen Anzahl von Geweben gezeigt, wie man ein Bild der Uber-alles-Funktion der Glycolyse im Sinne einer biochemischen "Gestalt" gewinnen kann. Enzymverteilungsmuster geben qualitativ die Richtung der Fließgleichgewichte an. Da die Bezie- 
hungen der Muster zum stationären Fluß und den in vivo Aktivitäten unter verschiedensten Bedingungen unïbersichtlich sind, haben wir zur Definition der Fließgleichgewichte den Weg über die Metabolit- und Flußmuster gewählt (Hess \& BRAND 1965,

Tabelle 2

Gleichungen für die Hin- und Rückflüsse

Die Reaktion

$$
[\mathrm{A}]+[\mathrm{B}] \underset{\mathrm{k}-1}{\stackrel{\mathrm{k}_{1}}{\underset{\mathrm{N}}{\longrightarrow}}}[\mathrm{C}]+[\mathrm{D}]
$$

ist im Massenwirkungsgleichgewicht definiert durch

$$
v_{1}=v_{-1} \quad v_{\text {net }}=0
$$

und

$$
K_{\mathrm{qpp}}=\frac{[\overline{\mathrm{C}}]\lfloor\overline{\mathrm{D}}]}{[\overline{\mathrm{A}}][\overline{\mathrm{B}}]}
$$

im stationaren Zustand durch

$$
v_{\text {net }}=v_{1}-v_{-1}
$$

und

$$
\Gamma=\frac{[C][D]}{[A][B]}
$$

da

$$
\begin{aligned}
& k_{\text {app }}\left(\frac{v_{-1}}{v_{1}}\right)=\Gamma \text { ist } \\
& v_{1}=\frac{v_{\text {net }}}{1-\Gamma / K_{\text {app }}} \text { and } \\
& v_{-1}=\frac{v_{\text {net }}}{k_{\text {app }} / \Gamma-1}
\end{aligned}
$$

Hess 1962, 1963). Die Metabolit-Spiegel können unter stationären Bedingungen und Verwendung des Nettoflusses zur Berechnung der Hin- und Rückflüsse jeder einzelnen glycolytischen Reaktion verwandt werden. Damit ist ein metabolischer Zustand quantitativ im Hinblick auf die Lage und Richtung der Fließgleichgewichte definiert.

Der Zusammenhang geht aus der Ableitung hervor, die in Tabelle 2 ausgeführt ist. Sie beruht auf der Reversibilität der Reaktionen und der Tatsache, daß die Aktivitäten aller an einem Umsatz beteiligten Enzyme im stationären Zustand identisch 
oder bei Verzweigungen einfach gebrochen beziehungsweise vervielfacht sind. Ein auf dieser Grundlage berechnetes Flußprofil ist auf Abbildung 2 dargestellt. Die Differenz der angegebenen Hin- und Rückflüsse ergeben jeweils den Nettofluß des Systems, der entsprechend der Mengenbilanz 27 beziehungsweise 54 Einheiten beträgt.

Man erkennt zwei Gruppen von Reaktionen: (1) Enzyme, die nahe dem Massenwirkungsgleichgewicht operieren $\left(\mathrm{v}_{1} / \mathrm{v}_{-1}\right.$ und dementsprechend $\mathrm{K}_{\mathrm{app}} / \Gamma$ nahezu 1$)$ und (2) Enzyme, die um Größenordnungen entfernt vom Gleichgewicht laufen. Bei der ersten Gruppe von enzymatischen Reaktionen handelt es sich um Energiekontrolle durch Massenwirkung. Man kann sagen, daß die Enzymkapazitäten ausreichen, um den Substratanfall von den entsprechenden Donor-Enzymen über das Flußgefälle zu bewältigen und damit sich selbst der Über-alles-Umsatzgeschwindigkeit anzupassen. Da die kinetischen Verhältnisse übersichtlich sind, lassen sich aus den vorliegenden Daten bei Kenntnis der Michaelis-Konstanten einfach die Maximalaktivitäten der Enzyme berechnen (Hess \& Brand 1965, Hess 1963). Sie stimmen bei Enolase, Phosphoglyceratmutase und Adenylatkinase mit den extrahierbaren Enzymaktivitäten überein.

Für die erhebliche Abweichung der zweiten Gruppe von Enzymen (vor allem der Kinasen) vom Massenwirkungsgleichgewicht müssen wir den Mechanismus der Zügelungskontrolle entweder durch Aktivierung oder Hemmung oder durch Kompartmentierung verantwortlich machen. Schon früher (Hess \& CHANCE 1961) haben wir gezeigt, daß die Phosphofruktokinase der Asciteszellen bei stationären Zuständen und Ubergangszuständen ihre Aktivität bei fallender ATP- und nahezu unveränderter F6P-Konzentration um ein Vielfaches erhöht. Damit und durch die Ausbildung eines kinetischen Kreuzungspunktes (BücHER 1961) war diese Reaktion als wichtiger Kontrollpunkt der Glycolyse ausgewiesen. Auch die anderen Enzyme dieser Gruppe - bei der Hefe muß Pyruvatdecarboxylase hinzugezählt werden - sind in diesem Sinne als Kontrollpunkte der glycolytischen Sequenz anzusehen. Auf die Beweisführung kann im einzelnen an dieser Stelle nicht eingegangen werden. Die Tatsache einer um, Größenordnungen vom Massenwirkungsgleichgewicht abweichenden Reaktion hat für die Identifizierung von Kontrollpunkten des Stoffwechsels größten heuristischen Wert (siehe unten) (Hess \& BRAND 1965).

Metabolit- und Flußprofile können als Grundlage eines mathematischen Modells der Glycolyse benutzt werden (Garfinkel \& Hess 1964). Unter Benutzung der Hinund Rückflüsse, der Michaelis-Konstanten und anderer bekannter kinetischer Konstanten lassen sich für jeden einzelnen enzymatischen Schritt die detaillierten Mechanismen ausarbeiten. Man bildet für jede einzelne Reaktion die entsprechenden Differentialgleichungen auf der Grundlage der Massenwirkungskinetik und simuliert katalytische Kontrollwirkungen durch Aktivatoren und Inhibitoren in Form von zusätzlichen „Konstanten“. Ein derartiges Gleichungssystem kann durch Digital-Rechenmaschinen mit einem einfachen numerischen Integrationsverfahren modifiziert nach EULER bearbeitet werden.

In Tabelle 3 ist eine Zusammenstellung der von uns berechneten Metabolit-Verhältnisse unter den Bedingungen einer stationären Glycolyse von EHRLICH-AscitesTumor-Zellen wiedergegeben. Man sieht, daß die berechneten und gemessenen Werte gut übereinstimmen (Garfinkel \& Hess 1964). 
Kontrollmechanismen der Glycolyse

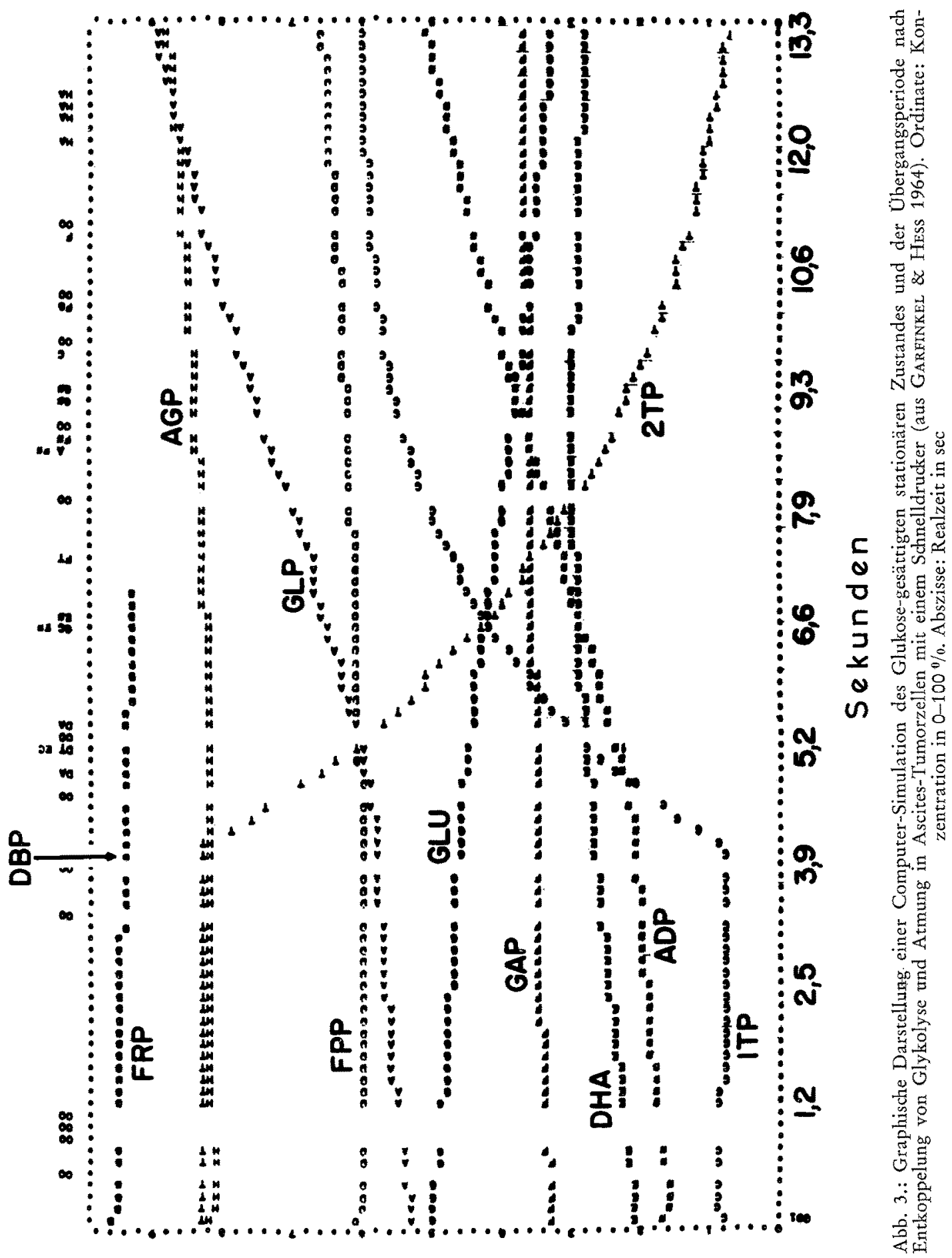


Abbildung 3 gibt schließlich eine graphische Darstellung einer Reihe von glycolytischen Intermediaten (Ordinate $=$ Konzentration in Prozent, Abszisse $=$ Realzeit) über einen stationären Zustand sowie nach Entkopplung durch Dibromphenol (DBP) wieder. Die Darstellung wurde durch den Schnelldrucker der Rechenmaschine (UNIVAC II) hergestellt. Man sieht, daß entsprechend dem beim PASTEUR-Effekt bekannten Übergangszustand der Verbrauch an Glucose (GLU) nach Entkopplung rasch ansteigt, zugleich der Gehalt an Adenosintriphosphat im Cytoplasma (1 TP) zu- und in den

\section{Tabelle 3}

Verhältnisse der Konzentration von Metaboliten in Ehrlich-Ascites-Tumor-Zellen und thre Computer-Berechnung. (Aus Garfinkel \& Hess 1964)

\begin{tabular}{|c|c|c|c|c|c|c|c|}
\hline \multirow{2}{*}{ Verhältnis } & \multicolumn{2}{|c|}{ 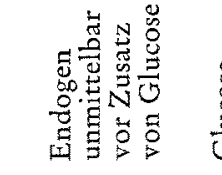 } & 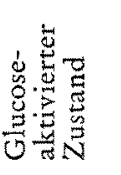 & \multicolumn{2}{|c|}{ 岕总 } & \multicolumn{2}{|c|}{ 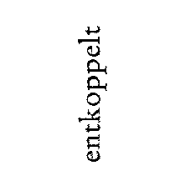 } \\
\hline & Comp. & Zelle & Comp. & Comp. & Zelle & Comp. & Zelle \\
\hline Lactat/Pyruvat & 152 & 164 & 138 & 77 & 69,5 & 179 & 135 \\
\hline $\begin{array}{l}\text { Dioxyacetonphosphat } \\
\alpha \text {-Glycerophosphat/Dihydroxy- }\end{array}$ & 1,23 & 1,25 & 3,64 & 9,25 & 11,6 & 9,44 & \\
\hline $\begin{array}{l}\text { aceton-Phosphat } \\
\text { Glyceraldehydphosphat/ }\end{array}$ & 8,62 & 9,1 & 7,15 & 6,31 & 7,8 & 6,29 & \\
\hline Dioxyacetonphosphat & 1,52 & 1,42 & 1,52 & 1,35 & 1,57 & 1,32 & \\
\hline ATP (cytoplasmatisch)/ADP & 3,64 & ca. 5 & 0,962 & 0,4 & & 1,18 & \\
\hline ATP (mitochondrial)/ADP & 0,41 & & 0,31 & 4,1 & & 0,14 & \\
\hline Gesamt-ATP/ADP & 4,05 & ca. 5,5 & 1,3 & 4,5 & & 1,3 & \\
\hline Fruktose 6-phosphat/Fruktose & & & & & & & \\
\hline 1,6-diphosphat & 0,188 & ca. 0,2 & 0,1 & 0,196 & 0,198 & ca. 0,1 & ca. 0,16 \\
\hline DPNI (cytoplasmatisch)/DPN & 0,062 & & 0,063 & 0,028 & & 0,068 & \\
\hline DPNH (mitochondrial)/DPN & 10,1 & & 1,97 & 0,294 & & 0,724 & \\
\hline Gesamt-DPNH/Gesamt-DPN & 0,648 & & 0,389 & 0,615 & & 0,246 & \\
\hline
\end{tabular}

Mitochondrien (2 TP) abnimmt und weiterhin Glucose-6-phosphat (GLP) sowie Fruktose-1,6-Diphosphat (FPP), Dioxyacetonphosphat (DHA) und schließlich Adenosintriphosphat (ADP) entsprechend den experimentellen Erfahrungen einem höheren stationären Spiegel zustreben. Die Untersuchung mathematischer Modelle durch Rechenmaschinen erlaubt, wie man sieht, nicht nur die Prüfung unserer Vorstellungen über stationäre Zustände, sondern auch der Obergänge des Stoffwechsels, die auf komplexere Kontrollwirkungen zurückzuführen und für unser Verständnis der Kontrollmechanismen von weitaus größerer Bedeutung sind.

\section{UBERGANGSZUSTANDE}

In Tabelle 4 sind verschiedene Ubergangsformen der Glycolyse seit der berühmten Beobachtung von PASTEUR (1876) zusammengestellt. In allen Fällen handelt es sich um die Reaktionen des glycolytischen Systems auf Anderung kontrollierender Para- 
meter. Jede Änderung führt zu einer Umstellung von Flüssen und stationären Metabolitkonzentrationen innerhalb von typischen Übergangszeiten zwischen 1 bis $30 \mathrm{sec}$, die für jedes glycolytische System charakteristisch sind. Die neu eingestellten stationären Zustände halten so lange an, wie die entsprechenden Parameter einwirken.

Tabelle 4

Übergangszustände der Glykolyse

\begin{tabular}{|c|c|c|c|}
\hline Zelle & Meßzustand & Wirkung & Autoren \\
\hline Hefe & stationär & $\begin{array}{l}\text { Hemmung von Gärung durch } \\
\text { Atmung }\end{array}$ & Pasteur (1876) \\
\hline Hefe & stationär & $\begin{array}{l}\text { Aktivierung der Gärung durch } \\
\text { Phosphat }\end{array}$ & Harden $\&$ Young $(1905)$ \\
\hline Muskel & stationär & Umkehr der Glykolyse & MEYERHOF (1920) \\
\hline $\begin{array}{l}\text { Ascites- } \\
\text { Tumorzelle }\end{array}$ & stationär & $\begin{array}{l}\text { Hemmung von Atmung durch } \\
\text { Glykolyse }\end{array}$ & Crabtree (1929) \\
\hline Hefe & stationär & $\begin{array}{l}\text { Kontrolle der Gärung durch } \\
\text { ADP und Phosphat }\end{array}$ & LYNEN (1941), JoHNSON (1941) \\
\hline Hefe & transient & $\begin{array}{l}\text { Aktivierung der aeroben } \\
\text { Glykolyse durch Glucose }\end{array}$ & Chance (1954) \\
\hline $\begin{array}{l}\text { Ascites- } \\
\text { Tumorzelle }\end{array}$ & transient & $\begin{array}{l}\text { Aktivierung der Atmung durch } \\
\text { Glucose }\end{array}$ & Chance \& Hess (1956) \\
\hline $\begin{array}{l}\text { Frosdh- } \\
\text { Sartorius- } \\
\text { Muskel }\end{array}$ & $\begin{array}{l}\text { transient- } \\
\text { stationär }\end{array}$ & $\begin{array}{l}\text { Aktivierung der Atmung durch } \\
\text { elektrische Erregung des } \\
\text { Muskels }\end{array}$ & Chance $\&$ Connelly $(1955)$ \\
\hline Hefe & transient & $\begin{array}{l}\text { Aktivierung der anaeroben } \\
\text { Glykolyse durch Glucose }\end{array}$ & Holzer (1952) \\
\hline Hefe & transient & $\begin{array}{l}\text { Oszillation der Pyridinnucle-- } \\
\text { otide }\end{array}$ & Duysens \& Amesz (1957) \\
\hline $\begin{array}{l}\text { Heuschrecken- } \\
\text { Flugmuskel }\end{array}$ & - stationär & $\begin{array}{l}\text { Aktivierung der Atmung durch } \\
\text { Glykolyse }\end{array}$ & BǘCHER (1961) \\
\hline $\begin{array}{l}\text { Ascites- } \\
\text { Tumorzelle }\end{array}$ & stationär & $\begin{array}{l}\text { Umkehr der oxydativen } \\
\text { Phosphorylierung }\end{array}$ & Hess (1962), Chance (1962) \\
\hline Herzmuskel & stationär & $\begin{array}{l}\text { Hemmung der Glykolyse durch } \\
\text { den Citratcyclus }\end{array}$ & $\begin{array}{l}\text { Garland, RANDLE \& NEWS- } \\
\text { HOLME }(1963), \text { WILLIAMSON } \\
(1964)\end{array}$ \\
\hline Hefe & $\begin{array}{l}\text { transient- } \\
\text { stationär }\end{array}$ & $\begin{array}{l}\text { Kontinuierliche Oszillation } \\
\text { der Glykolyse }\end{array}$ & $\begin{array}{l}\text { Hess, Pye \& BRAND (1966, } \\
1965)\end{array}$ \\
\hline
\end{tabular}

Die Untersuchung der Ubergangszustände liefert uns wesentliche Informationen über Kontrollpunkte, an denen Substanzen eingreifen und den Umsatz beschleunigen oder hemmen. Dem Regeltechniker sind verschiedenste Ubergangsformen technischer Systeme seit langem bekannt. In den vergangenen 10 Jahren konnten viele Übergangsformen auch in biochemischen Systemen nachgewiesen werden: Asymptotische Übergänge im Cytochromsystem (CHANCE \& Hess 1959), aperiodische Übergänge mit 
und ohne Umkehr in der Glykolyse und in der Zellatmung (Hess 1963, Chance \& Hess 1959, Chance 1961, Holzer \& Freytag-Hrlf 1959), schließlich oszillatorisch gedämpfte Ubergänge (Duysens \& Amesz 1957, Chance, Estabrook \& Goser 1964, Hommes 1964, Hess, Chance \& Betz 1964, Matrhal 1964, Betz \& Chance 1965). In letzter Zeit wurden schließlich erzwungene Schwingungen, überlagerte Oszillationen (Amplituden-Modulation), oszillatorisches "Aufschaukeln" sowie kontinuierliche Oszillationen (Hess \& Brand 1965, Hess, Pye \& Brand 1965) beobachtet. Die Untersuchung der stationär oder vorübergehend oszillierenden Glycolyse, besonders im zellfreien Extrakt, hat neue Einblicke in die Kontrollmechanismen der Glycolyse ergeben, die nun besprochen werden sollen.

\section{OSZILLIERENDE GLYCOLYSE}

Bei der oszillierenden Glycolyse handelt es sich um eine periodische Aktivierung und Hemmung der Glycolyse, wobei maximale Aktivierung im oberen Bereich der Glycolyse mit maximaler Hemmung im unteren Bereich der Glycolyse in einer Halbperiode und umgekehrt in der nächsten Halbperiode bei stationärer Alkoholproduktion abwechseln. Es handelt sich um eine Art von automatisch repetierender PAsTEURReaktion, bei der die durch Beschleunigung und Zügelung der Glycolyse entstehenden kinetischen Kreuzungspunkte periodisch durch die endogenen Eigenschaften des glycolytischen Systems unabhängig vom Beobachter produziert werden. Dementsprechend kommt es zu oszillierenden Anderungen der Konzentrationen der glycolytischen Intermediate über einen großen Bereich (80\% des cytoplasmatischen Pyridinnucleotids), deren Bewegung durch Registrierung der Pyridinnucleotide erkannt werden kann. Der Nettofluß durch das System ergibt sich aus dem Produkt von Amplitude (ausgedrückt in DPNH-Einheiten) und der Kreisfrequenz (Hess, Chance \& BeTz 1964), die kinetischen Kreuzungspunkte werden aus dem Phasendiagramm der Metabolite (BETZ \& Chance 1965) durch die entsprechende Phasenverschiebung identifiziert und können im zellfreien Extrakt durch Enzymtitrationen (Hess \& Brand 1966, Hess, Chance \& Betz 1964, Hess, Brand \& Cassuto 1965, Hess \& Brand 1965) bestätigt werden. Der Vorgang läuft in der intakten Zelle mit einer Frequenz von $1,7 \mathrm{~min}^{-1}$ bei $26^{\circ} \mathrm{C}_{3}$ im Extrakt mit einer Frequenz von $0,2 \mathrm{~min}^{-1}$ bei $25^{\circ} \mathrm{C}$ ab.

Abbildung 4 gibt einen. Ausschnitt aus der Registrierung der oszillierenden Glycolyse in einem zellfreien Extrakt aus Saccharomyces carlsbergensis wieder, der durch Ultraschallbehandlung und Ultrazentrifugation gewonnen war. Die Oszillation konnte 22. Stunden lang bei Zimmertemperatur mit konstanter Frequenz und einer Flußgeschwindigkeit von $9 \mathrm{Nanomol} \times \mathrm{min}^{-1} \times \mathrm{mg}^{-1}$ (Protein) unter Verwendung von Trehalose als Substrat mit einer Endkonzentration, von 0,34 M Alkohol nach 22 Stunden beobachtet werden (Hess \& Brand 1966, Hess, Pye \& Brand 1965). Der Q-Wert als Gütemaß der Oszillation (siehe Hess, Chance \& BeTz 1964) beträgt 1000 und liegt damit im Bereich elektronischer Oszillatoren. Aus diesem Experiment geht die außerordentliche Stabilität der Oszillationen und der daran beteiligten Enzyme hervor.

Die Analyse des zellfreien Extraktes ergibt alle bekannten Bestandteile des Enzymverteilungsmusters sowie des Metabolit-Musters von Bierhefen (Hess \& Brand 
1966, Hess, Chance \& Betz 1964). Da die Glucose-Konzentration im Extrakt zu vernachlässigen ist (kleiner als $10^{-6} \mathrm{~mol}$ ) und der Extrakt zunächst über Stunden ohne Substratzusatz oszilliert, muß endogenes Substrat zur Sättigung der Glycolyse vorliegen. Die Untersuchung auf glycogenartige Substrate ergab (Hess, Chance \& BeTz 1964) Aquivalente in der Größenordnung von $7 \mathrm{mM}$ (in Glucoseeinheiten). Da

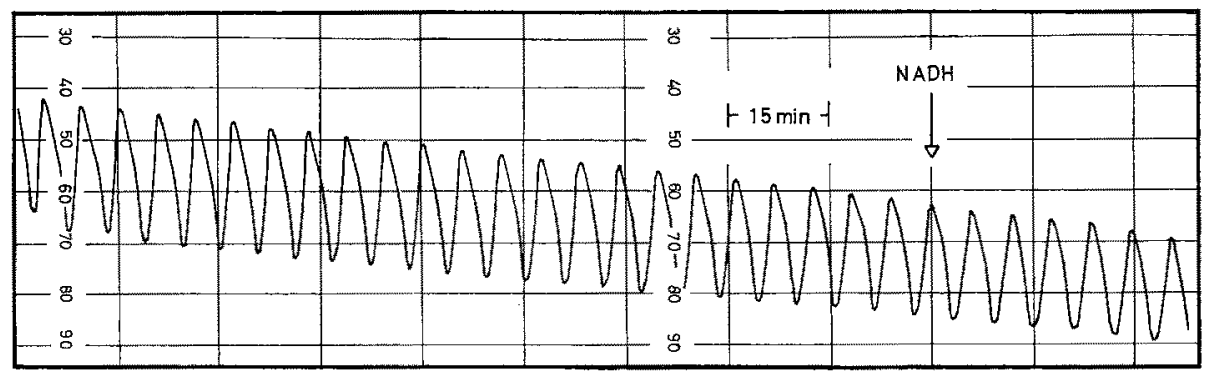

Abb. 4: Kontinuierliche Oszillation der Glykolyse in einem Extrakt von Saccharomyces carlsbergensis (Hess, Pye \& BRAND 1965). Messung der reduzierten Pyridinnucleotide in Fluorimeter "Eppendorf" $(366 / 400-3000 \mathrm{~m} \mu) \cdot \mathrm{d}=1 \mathrm{~mm}$. Temperatur $\sim 22^{\circ} \mathrm{C}$. Amplitudeneichung : $0,5 \mathrm{~cm}=131 \mathrm{nMol} \mathrm{NADH} / 200 \mu \mathrm{l}$

Trehalose (PyE 1965) ein gutes Substrat für die oszillierende Glycolyse ist, nehmen wir an, daß es sich zum Teil bei unseren Analysen um Trehalose-Einheiten handelt. Bei der Papierchromatographie wird nach orientierenden Versuchen neben Mannose Glucose frei (Hess, Pye \& Brand 1965).

Zur Aufklärung des Mechanismus der Oszillation haben wir das System mit reinen Gärungsenzymen titriert (Hess \& Brand 1965, Hess, Brand \& Cassuto 1965) und gefunden, daß Wellenformen und Frequenz auf Zusatz der Phosphokinasen empfindlich reagieren, während Zusatz von GAPDH, MK unter anderem erst bei etwa 15- bis 20 facher Steigerung der vorhandenen Aktivität den Ablauf beeinflussen. Abbildung 5 zeigt die Reaktion nach Zusatz einer PFK-Aktivität, die den vorhandenen Bestand etwa verdoppelt. Zunächst wurde eine nicht sinusförmige Wellenform beobachtet. PFK-Zusatz führt nach einer kurzen Reduktion zu einer vorzeitigen Umkehr der Periode in Richtung auf eine Oxydation des Systems, das schließlich sinusförmig auf einem stärker oxydierten mittleren Niveau weiterpendelt. Die Massenwirkung durch vermehrte ADP-Bildung drückt das System zur stärker oxydierten Seite. Die nun sinusförmige Wellenform führen wir auf die Aktivierung von PK durch phasenverfrühte Produktion von FDP zurück (siehe unten). Wie man weiter sieht, bleibt das Amplituden-Frequenz-Produkt konstant.

Ein Spiegelbildexperiment ist auf Abbildung 6 wiedergegeben. Hier führte die Verdoppelung der PGK-Aktivität zu einer raschen Verschiebung des Systems zu einem mehr reduzierten Zustand, dessen stöchiometrischer Zusammenhang auf dem Blockdiagramm veranschaulicht ist. Der nicht sinusförmige Verlauf führt wieder zum sinusförmigen Verlauf. Das Amplituden-Frequenz-Produkt bleibt ebenfalls konstant (die Experimente auf Abbildungen 5a und 6a sind absolut spiegelbildlich, da die Registrierung auf Abbildung 6a mit halber Empfindlichkeit vorgenommen wurde). Die rasche 
Reduktion des Systems führen wir auf eine Enthemmung von GAPDH durch Wegnahme von 1,3-DPGA zurïck, eine Folgerung, die nach Kontrollexperimenten durch Titration mit ATP beziehungsweise 1,3-DPGA bestätigt werden konnte (HEss, Brand

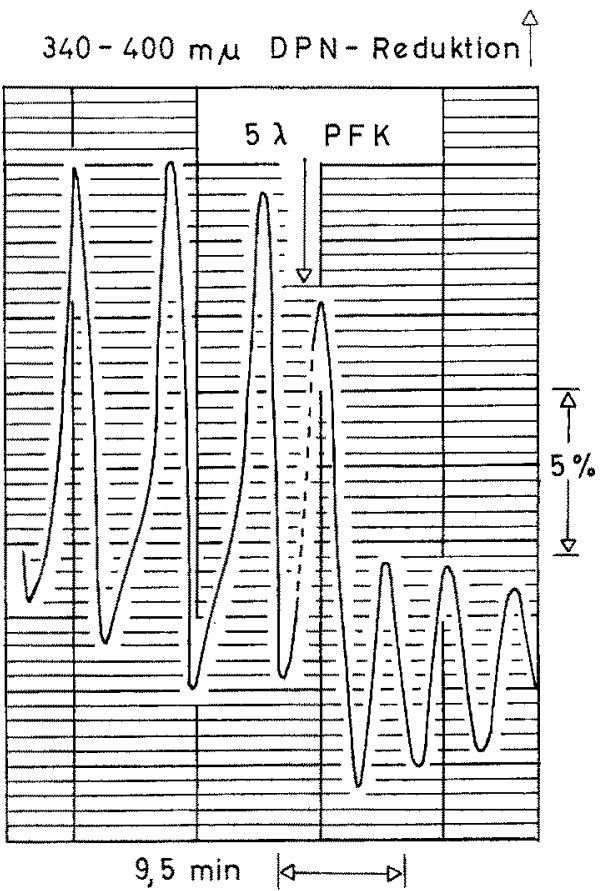

Abb. 5a: Titration der oszillierenden Glykolyse durch PFK. $\mathrm{d}=1 \mathrm{~mm}$, Temperatur $\sim 22^{\circ} \mathrm{C}$. Ordinate in $\%$ Extinktionsdifferenz

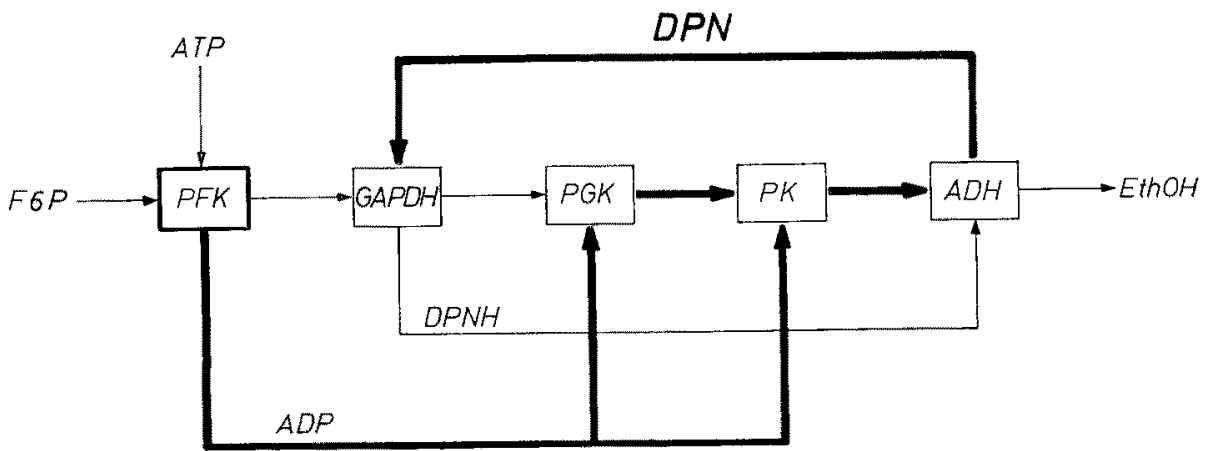

Abb. 5b: Blodkdiagramm der durch die Titration erzeugten stöchiometrischen Verschiebungen

\& CAssuto 1965). Das System reagiert ebenfalls empfindlich auf Zugabe von PK durch Verkleinerung von Amplitude und Erhöhung der Frequenz, jedoch tendiert das System zur stärker oxydierten Seite, die vor allem durch die ATP-Hemmung von HK und PFK bestimmt wird (siehe unten). 
Aus diesen Experimenten sowie aus Metabolit-Titrationen (CHANCE, Schoener \& ElSAESSER 1964) geht die Bedeutung des ATP/ADP-Systems für die Kontrolle der Oszillation hervor. Auf Grund der Metabolit-Messungen und der Titrationsexperimente ergibt sich ein Zusammenhang, der für den sinusförmigen Verlauf durch das Phasenwinkel-Diagramm auf Abbildung 7 demonstriert wird. Für den nicht sinus-

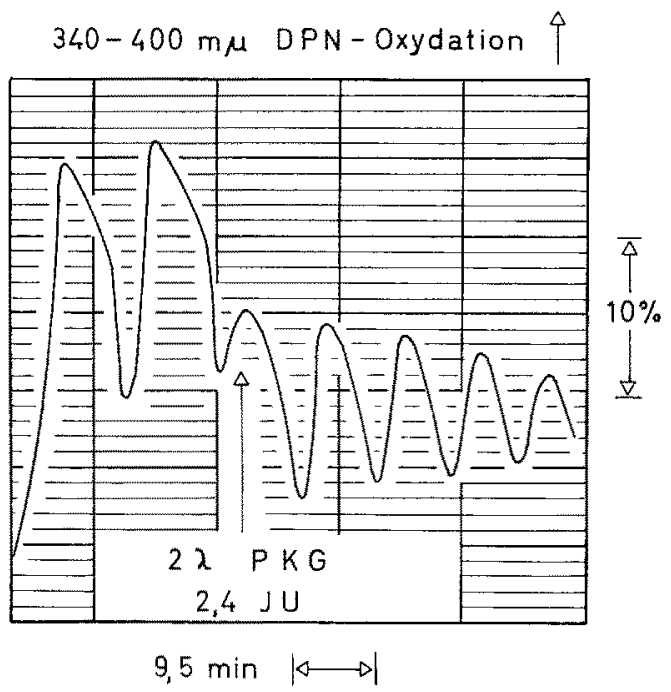

Abb. 6a: Titration der oszillierenden Glykolyse durch PGK. $d=1 \mathrm{~mm}$, Temperatur $\sim 22^{\circ} \mathrm{C}$. Ordinate in $\%$ Extinktionsdifferenz

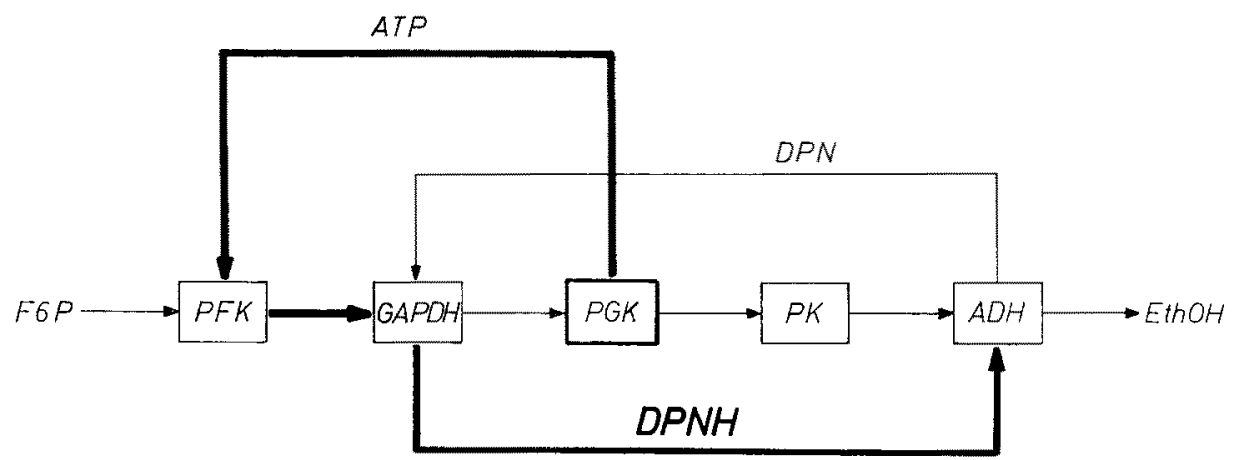

Abb. 6b: Blockdiagramm der durch die Titration erzeugten stöchiometrischen Verschiebungen

förmigen Verlauf ist die Stellung von FDP um etwa $90^{\circ}$ verzögert. Dem Phasendiagramm der Metabolit-Konzentrationen entspricht das Phasendiagramm aller an der Glycolyse beteiligten Enzymaktivitäten. Die Phasenlage der für die Kontrolle der Oszillation wesentlichen Enzymaktivitäten ist schematisch dargestellt.

Wenn sich auch aus der Phasenlage die kinetischen Kreuzungspunkte des Systems klar ergeben, so sind damit die für dic Oszillation entscheidenden Dämpfungs- bezie- 
hungsweise Verstärkungskomponenten noch nicht bezeichnet. Die Reaktion des Systems sowie der Konzentrationsbereich identifizieren ATP eindeutig als Dämpfungskomponente. Die Phosphokinasen werden wenigstens über $90^{\circ}$ der Phase von ATP gehemmt (Hess, Pye \& BRAND 1965). Die Reaktion der Kinasen auf ADP ist unabhängig von
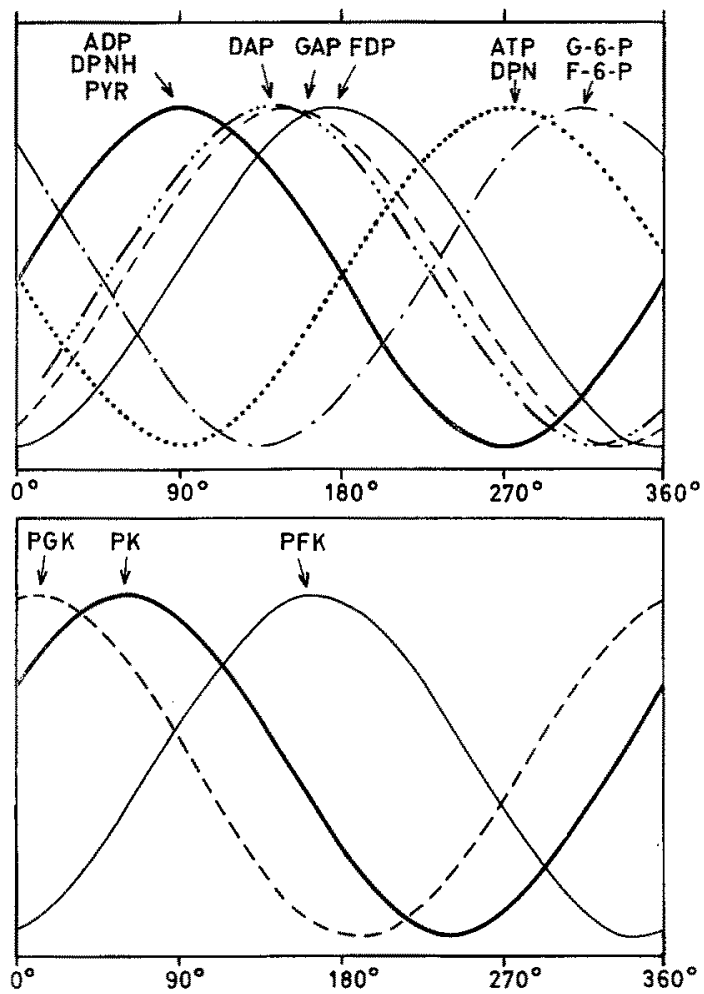

Abb. 7: Phasenwinkeldiagramm der Konzentration von glykolytischen Zwischenstoffen sowie der Aktivitäten von PGK, PFK und PK

seiner Massenwirkung wenig eindeutig. Wir haben daher nach einer anderen Verstärkungskomponente gesucht und gefunden, daß PK von Hefe (nicht aus der Muskulatur) im physiologischen Bereich stark durch FDP aktiviert wird, wie aus Abbildung 8 (HEss \& BRAND 1965) hervorgeht.

Damit können wir ein Kontrollnetz entwerfen (siehe Abbildung 9), das uns erklärt, wie durch ATP-Rückkopplung und FDP-Vorwärtskopplung die Glycolyse über einen großen Konzentrations- und Aktivitäts-Bereich hin- und herpendelt, wobei Energie und Aktivitätskontrolle nebeneinander wirksam sind. Das auf Abbildung 9 dargestellte Kontrollnetz entspricht formal, jedoch nicht spezifisch, dem von HrGGINs (1964), das auf einer gekreuzten Rückkopplung beruht und mit einem Analog-Computer-System simuliert wurde. Unklar in diesem Netz sind die Aktivierungskomponenten von PFK und HK, die bislang nicht eindeutig identifiziert werden konnten. 


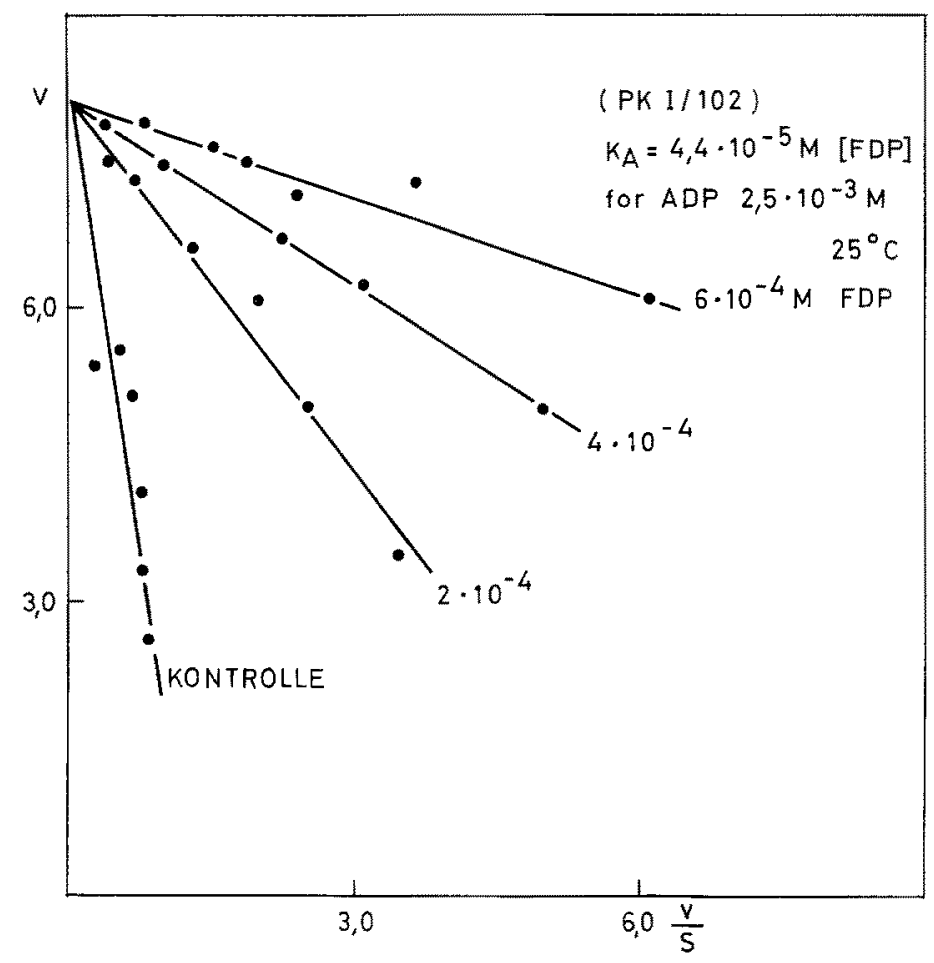

Abb. 8: Aktivierung der Hefepyruvatkinase durch FDP

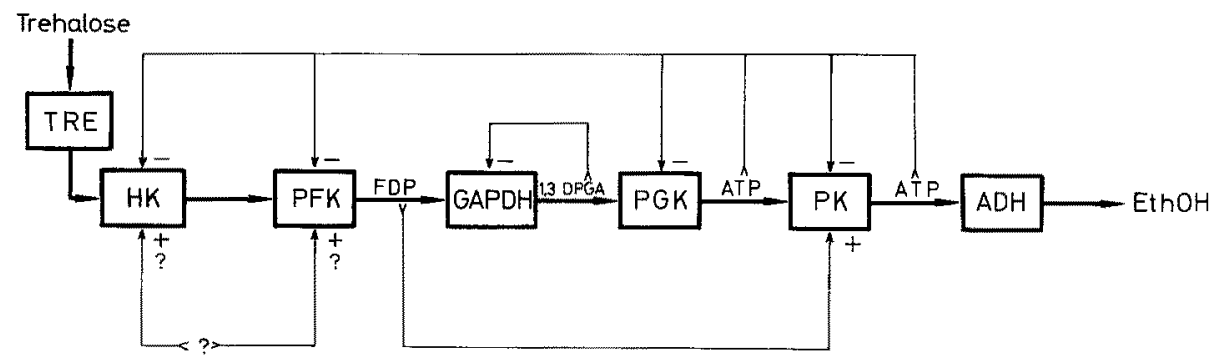

Abb. 9: Kontrollnetz der oszillierenden Glykolyse

\section{AUSBLICK}

Überblickt man die heutigen Vorstellungen der Kontrolle der Glycolyse, so erkennt man, daß der Umsatz dieses elementaren Prozesses des Zellstoff wechsels nicht allein durch Massenwirkung, sondern und vor allem durch Aktivitätskontrolle festgelegt wird. Die Rolle des klassischen Kontrollenzymes der Glycolyse GAPDH ist zurückgetreten. PFK, auf dessen Bedeutung LARDY (1956) zuerst hingewiesen hat, und die anderen Kinasen haben weitaus größere Bedeutung als Kontrollenzyme. Sie be- 
stimmen den Ablauf der oszillierenden Glycolyse, die als das Modell eines allgemeinen biochemischen Uhrenmechanismus dienen kann. Erstaunlich ist die Wirkung von ATP, deren Struktur Lohmann vor über 30 Jahren entdeckt hat und deren Funktion als Energieüberträger und, wie man erst jetzt erkennt, als Kontrollmetabolit von universeller Bedeutung ist.

\section{ZUSAMMENFASSUNG}

1. Die Mechanismen der Kontrolle enzymatischer Reaktionssequenzen lassen sich allgemein auf Wechselwirkungen von Aktivatoren, Inhibitoren, Substraten und Produkten mit Enzymproteinen sowie auf Induktion oder Repression der Enzymsynthesen zurückführen. Alle Kontrolltypen werden im Verlaufe der Glycolyse beobachtet. Sie sind die Grundlage des Kontrollnetzes, das den Ablauf der Glycolyse bestimmt und nach dem Rückkopplungsprinzip operiert.

2. Das stationäre Verhalten der Fließgleichgewichte der Glycolyse kann durch Bestimmung von Netto-Fluß und stationären Intermediatkonzentrationen adequat in Form der Metabolit- und Flußprofile für den Hin- und Rückfluß jeder enzymatischen Reaktion beschrieben werden. Derartige Profile kennzeichnen den Kontrolltyp jeder enzymatischen Reaktion. Metabolit- und Flußprofile können als Grundlage mathematischer Modelle der Glycolyse benutzt werden. Das Verhalten dieser Modelle unter stationären und transienten Bedingungen steht in Ubereinstimmung mit den experimentellen Beobachtungen.

3. Die Untersuchung von Übergangszuständen ergänzt die Analyse stationärer $\mathrm{Zu-}$ stände. Sie führt unabhängig zur Aufdeckung von Kontrollpunkten der glycolytischen Sequenz und erfaßt allgemein den Bereich sowie die Güte biochemischer Kontrollmechanismen.

4. Der Mechanismus der stationär oder transient oszillierenden Glycolyse konnte im zellfreien Extrakt durch Metabolitanalysen, durch Titrationen mit Intermediaten und Enzymen weitgehend aufgeklärt werden. Er beruht auf der spezifischen Kontrolle der Phosphotransferasen durch gekreuzte Rückkopplung und stellt das biochemische Modell zellulärer Uhrenmechanismen dar.

Ausgearbeitet nach den Vorträgen auf dem II. Internationalen Symposion über quantitative Biologie des Stoffwechsels der Biologischen Anstalt Helgoland sowie der II. Jahrestagung der Arbeitsgemeinschaft "Biochemie" in der Gesellschaft für experimentelle Medizin, Magdeburg 1965.

Wir danken der Deutschen Forschungsgemeinschaft Bad Godesberg bei Bonn, dem US Public Health Service Bethesda. MD. sowie der Research Corporation New York für die großzügige Unterstützung unserer Arbeiten.

\section{ZITIERTE LITERATUR}

Betz, A. \& Chance, B., 1965. Influence of inhibitors and temperature on the oscillation of reduced pyridine nucleotides in yeast cells. Archs Biochem. Biophys. 109, 579-584. 
- 1965. Phase relationship of glycolytic intermediates in yeast cells with oscillatory metabolic control. Archs Biochem. Biophys. 109, 585-594.

BücHER, T., 1961. Intermediärstoff wechsel der Glucose unter zellphysiologischem Aspekt. In: Die endokrine Behandlung des Mamma- und Prostatacarcinoms. - Endokrine Regulationen des Kohenhydratstoffwechsels. 7. Symposium d. Dt. Ges. f. Endokrinologie, Homburg, Saar, 1960. Hrsg. von H. Nowakowski. Springer, Berlin, 129-134.

Chance, B., 1953-54. Enzymes in action in living cells: the steady-state of reduced pyridine nucleotides. In: The Harvey lectures. Acad. pr., New York, Ser, 49, 145-175.

- 1961. Control characteristics of enzyme systems. Cold Spring Harb. Symp. quant. Biol. 26, 289-299.

- 1962. Symposium Löwen, Juni 1962.

- \& Hess, B., 1956. On the control of metabolism in ascites tumor cell suspensions. Ann. N.Y. Acad. Sci. 63, 1008-1016.

- \& Hess, B., 1959. Spectroscopic evidence of metabolic control. Science, N.Y. 129, 700-708.

- Conelly, C. M. \& Hess, B., 1955. The respiratory chain as an indicator of intracellular ADP levels in muscle, yeast and tumor cells. J. cell. comp. Pbysiol. 46, 358.

- Estabrook, R. W. \& Gosh, A., 1964. Damped sinusoidal oscillations of cytoplasmic reduced pyridine nucleotides in yeast cells. Proc. natn. Acad. Sci. U.S.A. 51, 1244-1251.

- Schoentr, B. \& Elsaesser, S., 1964. Control of the waveform of oscillations of the reduced pyridine nucleotide level in a cell-free extract. Proc. natn. Acad. Sci. U.S.A. 52, 337-341.

CRABTREE, H. G., 1929. Observations on the carbohydrate metabolism of tumors. Biocbem. J. 23, 536 .

Duysens, L. N. M. \& AMEsz, J., 1957. Fluorescence spectrophotometry of reduced pyridine nucleotide in intact cells in the near ultraviolet and visible light. Biochim. biophys. Acta 24, 19-26.

Eigen, M. \& Hammes, G. G., 1963. Elementary steps in enzyme reactions. Adv. Enzymol. 25, $1-38$.

Garfinkel, D. \& Hess, B., 1964. Metabolic control mechanism. 7. A detailed computer model of the glycolytic pathway in ascites cells. J. biol. Chem. 239, 968-971.

Garland, P. B., Randle, P. J. \& Newsholme, E. A., 1963. Citrate as an intermediary in the inhibition of phosphofructokinase in rat heart muscle by fatty acids, ketone bodies, pyruvate, diabetes and starvation. Nattre, Lond. 200, 169-170.

Harden, A. \& YounG, W. I., 1905. The influence of phosphate on the fermentation of glucose by yeast-juice. J. chem. Soc. $\mathbf{8 7}, 189$.

Hess, B., 1962. Control of metabolic rates. J. gen. Physiol. 45, 603 A.

- 1963. Control of metabolic rates: Regulation of metabolism. In: Control mechanisms in respiration and fermentation. 8th Annual symposium of the Soc. of Gen. Physiol., Woods Hole, Mass., 1961. Ed. by B. E. Wright. Ronald, New York, 333.

- 1963. Fließgleichgewicht der Zellen. Dt. med. Wschr. 88, 668-676.

- 1963. Koordination von Atmung und Glykolyse. In: Funktionelle und morphologische Organisation der Zelle. Wiss. Konf. d. Ges. Dt. Naturf. u. Arzte, Rottach-Egern, 1962. Hrsg. von P. Karlson. Springer, Berlin, 163-185.

- 1963. Reactions of respiratory components in intact cells. In: Intracellular respiration: Phosphorylating and non-phosphorylating oxidation reactions. Ed. by E. C. Slater. Oxford, Pergamon pr. (Proc. 5th Int. Congr. Biochem., Moscow 5, 313.)

- \& Brand, K., 1965. Enzyme and metabolite profiles. In: Control of energy metabolism. Ed. by B. Chance. Acad. pr., New York, 111-122.

- 1965. Mechanism of glycolytic oscillation. In: Abstracts of papers presented at the 150th Meeting of the Am. Chem. Soc., $27 \mathrm{c}$.

- - 1966. Continuous glycolytic oscillations in extracts of $S$. carlsbergensis. Fedn Proc. Fedn Am. Socs exp. Biol. (Abstr.)

- - \& CAssuto, Y., 1965. Enzyme and metabolite pattern in the oscillating cell-free extract of S. carlsbergensis. Fedn Proc. Fedn Am. Socs exp. Biol. 24, 537.

- \& ChANCE, B., 1959. Ober zelluläre Regulationsmechanismen und ihr mathematisches Modell. Naturwissenschaften 46, 248-257. 
- - 1961. Metabolic control mechanism. 6. Chemical events after glucose addition to ascites tumor cells. J. biol. Chem. 236, 239-246.

- - \& BETz, A., 1964. Isolierung eines oszillierenden Systems aus S. carlsbergensis. Ber. Bunsenges, physikal. Chem. 68, 768.

- PYE, K. \& BRAND, K., 1965. Unpublizierte Untersuchungen.

Higgins, J., 1964. A chemical mechanism for oscillation of glycolytic intermediates in yeast cells. Proc. natn. Acad. Sci. U.S.A. 51, 989-994.

Holzer, H., 1952. München, Naturwiss. Hab.-Schr. v. 1952.

- \& FREYTAG-Hilf, R., 1959. Zusammenwirken der Gärungsenzyme beim anaeroben und aeroben Glucoseumsatz in Hefezellen. Hoppe-Seyler's Z. physiol. Chem. 316, 7-30.

Hommes, S. A., 1964. Oscillatory reductions of pyridine nucleotides during anaerobic glycolysis in brewers yeast. Archs Biochem. Biophys. 108, 36-46.

Jornson, M. J., 1941. The role of aerobic phosphorylation in the Pasteureffect. Science, N.Y. 94, 200-202.

Lardy, H. \& Parks, R. E., 1956. Influence of ATP concentration on rates of some phosphorylation reactions. In: Enzymes: Units of biological structure and function. Ed. by $\mathrm{O} . \mathrm{H}$. Gaebler. Acad. pr., New York, 584-587.

LXNEN, F., 1941. Uber den aeroben Phosphatbedarf der Hefe. Justus Liebigs Annln Chem. 546, $120-141$.

- Hartmann, G., Netter, K. \& Schuegraf, A., 1959. In: The Regulation of cell metabolism. Ed. by G. E. W. Wolstenholme \& C. M. O'Connor. Little, Brown \& Co., Boston, Mass., 256. (CIBA Foundation Symposium.)

Matтhäı, J. M., 1964. Persönliche Mitteilung.

Meyerhof, O., 1920. Uber die Energieumwandlungen im Muskel. Pflügers Arch. ges. Physiol. $182,284-317$.

Monod, J., Changeux, J.-P. \& Jacobs, F., 1963. Allosteric proteins and cellular control systems. J. molec. Biol. 6, 306-329.

Parmiggianni, A., Love, D. S. \& Krebs, E. G., 1964. Purification and properties of rabbit muscle phosphofructokinase. Fedn Proc. Fedn Am. Socs exp. Biol. 23, 533, 2591.

Pasteur, L., 1876. Etudes sur la bière. Bull. Soc. chim. Paris, 240.

PetTE, D., 1965. Plan und Muster im zellulären Stoffwechsel. Naturwissenschaften 52, 597-616.

PYE, K., 1965. Unveröffentl. Befunde.

Williamson, J. R., Jones, W. A. \& Azzone, G. F., 1964. Metabolic control in perfused rat heart during fluoroacetate poisoning. Biochem. biophys. Res. Commun. 17, 696-702.

\section{Diskussion im Anschluß an den Vortrag Hess \& BRAND}

AEBI: Diese wichtigen Untersuchungen eröffnen verheißungsvolle Perspektiven. Wenn schon zellfreie und organellfreie Hefeextrakte zum Oszillieren gebracht und durch Substrat- beziehungsweise Enzymzusätze gestartet oder phasenverschoben werden können, so erscheint prinzipiell auch die Möglichkeit gegeben, oszillierende Systeme durch geeignetes Mischen von Substrat und Reinenzymen "synthetisch" darzustellen. Sind bereits Versuche in dieser Richtung unternommen worden? Lassen sich derartige Oszillationen auch an geeigneten Präparaten aus Warmblütergewebe (z. B. Muskelextrakt) erzeugen? Welches ist die Temperaturabhängigkeit dieser Oszillation? Ließe sich aus Experimenten, die "vorschriftsgemäß" bei $25^{\circ}$ beziehungsweise $30^{\circ} \mathrm{C}$ ausgeführt worden sind, ohne weiteres auf die Situation in vivo schließen?

Hess: The frequency of the oscillation is temperature dependent with an activation energy of $19 \mathrm{kcal} \times \mathrm{Mol}^{-1}$. The frequency is $0,56 \mathrm{~min}^{-1}$ at $35^{\circ} \mathrm{C}$. We are preparing all components which, to our knowledge, are necessary for the oscillation and hope to be able to synthesize an oscillating system by appropriate mixing of substrates and enzymes. Oscillation has recently been observed in extract of bovine heart muscle.

HeInMETs: I should like to mention that in addition to oscillatory phenomena in enzyme activ- 
ity, theoretical analyses of certain genetic schemes (JACOB \& MONOD 1961) also reveal oscillatory enzyme formation.

Marmasse: It is of interest to note that other types of oscillations exist. For instance, oscillations of redox-potential can sometimes be recorded in suspensions of cell-free homogenates of rat hepatoma or in dilutions of human blood. It must be pointed out that such oscillations were not consistently observed; their frequency ranged from 10 to 30 per minute approximately; they were obtained in a well buffered media with electrodes of polished platinum or of pure gold, in an inert atmosphere (nitrogen or argon).

Hess: I understand there are a number of systems which show oscillations. The problem is only how well can they biochemically be defined.

RoBERTS: You have mentioned the oscillatory mechanism in yeast cells, but in multicellular systems, how might the oscillatory activity between cells be linked?

Hess: At the present state it is very difficult to make any comments on the coupling of oscillatory activities between various cells. 